\title{
A deteriorating inventory model for quadratic demand and constant holding cost with partial backlogging and inflation
}

\author{
Dr. Ravish Kumar Yadav , Amit Kumar vats \\ (Associate Professor, Department of Mathematics, Hindu college ,Moradabad) \\ (Department of Applied Science ABES Group of Institutions Ghaziabad)
}

\begin{abstract}
In most of the deteriorating items inventory model, demand rate has considered as a constant function. But in realistic situation these cost varying according to time. So in this paper, we developed a deterministic deteriorating inventory model with inflation in which demand rate are quadratic function of time, deterioration rate is constant, backlogging rate is variable and depend on the length of the next replenishment, shortages are allowed and partially backlogged. The model is solved numerically by minimizing the total inventory cost for cycle.
\end{abstract}

Keywords: inventory, deteriorating items, shortages, time dependent demand, partial backlogging, constant holding cost and inflation.

\section{Introduction}

Buzacott (1975) developed the first EOQ model taking inflationary effects into account. In this model, a uniform inflation is assumed for all the associated costs and an expression for the EOQ is derived by minimizing the average annual cost. At most the same time Misra (1975) developed an economic order quantity model taking inflationary effects on inventory systems into account. In this model, a uniform inflation rate for all the costs is assumed and an expression for the EOQ is derived by minimizing the average annual cost. Misra (1979) then extended the EOQ model with different inflation rates for various associated costs. Today, inflation has become a permanent feature of the economy. Many researchers have shown the inflationary effect on inventory policy. Chang (2004) study EOQ model with deteriorating items under inflation when supplier credits linked to order quantity.Misra (1979) given a note on optimal inventory management under inflation. Yang (2004) work on inventory models for deteriorating items with shortages under inflation .Yang (2006) given Two-warehouse partial backlogging inventory models for deteriorating items under inflation

Deterioration is a term now commonly used in health care, to describe worsening of a patient's condition. It is often used as a shortened form of 'deterioration not recognized or not acted upon'. Much work to reduce harm from deterioration has been undertaken by the National Patient Safety Agency. It is the process in which an item loses its utility and become useless. Inventory in deteriorating items is a general phenomenon in daily life. The items like milk, fruit, vegetables, fashion goods, electronic components, eggs, medicines, wine and gasoline etc. are called deteriorating items. So deterioration of physical goods in stock is a very realistic factor and there is a big need to consider this in inventory model.

Harris (1915) developed first inventory model, Economic Order Quantity, which has generalized by Wilson (1934) and he gave a formula to obtain economic order quantity. Inventory of deteriorating items first studied by Whit-in (1957), he considered the deterioration of fashion goods at the end of prescribed storage period. Ghare and Schrader (1963) extended the classical EOQ formula with exponential decay of inventory due to deterioration and gave a mathematical model of inventory of deteriorating items. Dave and Patel (1981) developed the first deteriorating inventory model with linear trend in demand. He considered demand as a linear function of time. Chang and Dye (1999) developed an inventory model with time varying demand and partial backlogging. Goyal and Giri (2001) gave recent trends of modeling in deteriorating items inventory .They classified inventory models on the basis of demand variations and various other conditions or constraints. Ouyan get al. (2005) developed an inventory model for deteriorating items with exponential declining demand and partial backlogging. Dye et al.(2007). Find an optimal selling price and lot size with a varying rate of deterioration and exponential partial backlogging. They assume that a fraction of customers who backlog their orders increases exponentially as the waiting time for the next replenishment decreases. Alamri and Balkhi (2007) studied the effects of learning and forgetting on the optimal production lot size for deteriorating items with time varying demand and deterioration rates. Dye (2007) gave an inventory model to determining optimal selling price and lot size with a varying rate of deterioration and exponential partial backlogging and deterministic inventory model for deteriorating items with capacity constraint and timeproportional backlogging rate. Teng et al. (2007) gave a comparison between two pricing and lot sizing models with partial backlogging and deteriorated items. Roy (2008) developed an inventory model for deteriorating items with time varying holding cost and demand is price dependent. Liao (2008) aver an EOQ model with non 
instantaneous receipt and exponential deteriorating item under two level trade credits. Pareek and Kumar (2009) developed a deterministic inventory model for deteriorating items with salvage value and shortages. S.kouri et al. (2009) developed an Inventory models with ramp type demand rate, partial backlogging and Weibell's deterioration rate. Shah and Shukla (2009) developed a deteriorating inventory model for waiting time partial backlogging when demand and deterioration rate is constant. They made Abad $(1996,2001)$ more realistic and applicable in practice. He et al. (2010) gave an optimal production inventory model for deteriorating item with multiple market demand. Biswaranjan-Mandal (2010) gave an EOQ inventory model for Weibull distributed deteriorating items under ramp type demand and shortages. Yadav and Devi (2013).Develop of an inventory model with volume flexibility, random deterioration and increasing demand exponentially. Yadav and Yadav, (2013).work on Volume flexibility in production model with cubic demand rate and weibull deterioration with partial backlogging .Yadav, and Trivedi,(2013) study on a multi -echolen inventory models for deteriorating with partial backlogging.

In this paper, we developed a deterministic deteriorating inventory model with inflation in which demand rate are quadratic function of time, deterioration rate is constant, backlogging rate is variable and depend on the length of the next replenishment, shortages are allowed and partially backlogged. The model is solved numerically by minimizing the total inventory cost for cycle.

\section{Notation and Assumptions}

The mathematical model is based on the following notations and assumptions. Following notation are used in paper.

$\mathrm{C}_{\mathrm{o}} \quad$ the ordering cost per order;

$\mathrm{C}_{\mathrm{P}} \quad$ the purchase cost per unit;

$\theta$ the deterioration rate;

$\mathrm{C}_{\mathrm{H}}(\mathrm{t}) \quad$ the inventory holding cost per unit per time unit;

$\mathrm{C}_{\mathrm{B}} \quad$ the backordered cost per unit short per time unit;

$\mathrm{C}_{\mathrm{L}} \quad$ the cost of lost sales per unit;

$\mathrm{t}_{1} \quad$ the time at which the inventory level reaches zero $t_{1} \geq 0$.

$t_{2} \quad$ the length of period during which shortages are allowed, $t_{2} \geq 0$;

$\mathrm{T}$ the length of cycle time;

$I_{\max } \quad$ the maximum inventory level during $[0, \mathrm{~T}]$;

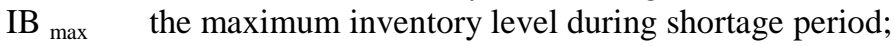

$\mathrm{Q}_{0} \quad\left(\mathrm{I}_{\max }+\mathrm{IB}_{\max }\right)$ the order quantity during a cycle of length $\mathrm{T}$;

$\mathrm{I}_{\mathrm{P}}(\mathrm{t}) \quad$ the positive inventory level at time $\mathrm{t}$;

$\mathrm{I}_{\mathrm{N}}(\mathrm{t}) \quad$ the negative inventory level at time $\mathrm{t}$;

$\mathrm{T}_{\mathrm{C}}\left(\mathrm{t}_{1}, \mathrm{t}_{2}\right)$ the total cost per time unit.

\section{Assumptions}

The model is based on the following assumptions

1. The demand rate is time dependent that is if ' $a$ ' is fix fraction of demand and ' $b$ ' and 'c' are that fraction of demand which is vary with time then demand function is $\mathrm{D}(\mathrm{t})=\mathrm{a}+\mathrm{bt}+\mathrm{ct}^{2}$, where $\mathrm{a}>0, \mathrm{~b}>0, \mathrm{c}$ $>0$.

2. The inflation rate is $\mathrm{r}(\mathrm{t})=\mathrm{e}^{-\mathrm{rt}}, \mathrm{r}>0$.

3. Holding cost is constant $\mathrm{h}(\mathrm{t})=\mathrm{h}$.

4. Shortages are allowed and partially backlogged.

5. The lead time is zero.

6. The replenishment rate is infinite.

7. The planning horizon is finite.

8. The deterioration rate is constant.

9. During stock out period, the backlogging rate is variable and is dependent on the length of the waiting time for next replenishment. So that the backlogging rate for negative inventory is, $\mathrm{R}_{\mathrm{B}}(\mathrm{t})=\frac{1}{1+\delta(T-t)}$ where $\boldsymbol{\delta}$ is backlogging parameter and $(\mathrm{T}-\mathrm{t})$ waiting time $\left(\mathrm{t}_{1} \leq \mathrm{t} \leq \mathrm{t}_{2}\right)$.

\section{Mathematical Formulation of Model}

The rate of change of inventory during positive stock period $\left[0, t_{1}\right]$ and shortage period $\left[t_{1}, T\right]$ is governed by the differential equations.

$$
\frac{d I_{P}}{d t}+\theta I_{P}=-\left(\mathrm{a}+\mathrm{bt}+\mathrm{ct}^{2}\right), \quad 0<\mathrm{t} \leq \mathrm{t}_{1}
$$


$\frac{d I_{N}}{d t}=\frac{-1}{1+\delta(T-t)}\left(\mathrm{a}+\mathrm{bt}+\mathrm{ct}^{2}\right), \mathrm{t}_{1} \leq \mathrm{t} \leq \mathrm{T}$

With boundary conditions $\mathrm{I}_{\mathrm{P}}(\mathrm{t})=\mathrm{I}_{\mathrm{N}}(\mathrm{t})_{=} 0$ at $\mathrm{t}=\mathrm{t}_{1}$ and $\mathrm{I}_{\mathrm{P}}(\mathrm{t})_{=} \mathrm{I}_{\max }$ at $\mathrm{t}=0$.

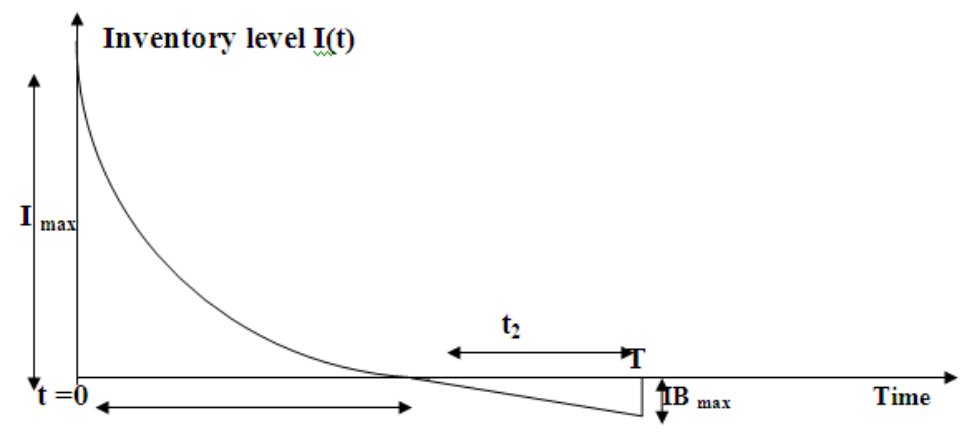

$\mathbf{t}_{1}$

Figure1 of inventory system

\section{Analytical Solution}

Case.1.The Inventory level have not shortages:

In this period $\left[0, t_{1}\right]$, the inventory depletes due to the deterioration and demand. So the inventory level at the time during $\left[0, \mathrm{t}_{1}\right]$ form the differential equation:

$$
\frac{d I_{P}}{d t}+\theta I_{P}=-\left(\mathrm{a}+\mathrm{bt}+\mathrm{ct}^{2}\right), 0<\mathrm{t} \leq \mathrm{t}_{1}
$$

With boundary conditions $I_{P}(t)=0$ at $t=t_{1}$.

The system gives the solution:

$$
\mathrm{I}_{\mathrm{P}}=e^{\theta\left(t_{1}-t\right)}\left\{\frac{a}{\theta}+\frac{b\left(\theta t_{1}-1\right)}{\theta^{2}}+\frac{c\left[\left(\theta t_{1}-1\right)^{2}+1\right]}{\theta^{3}}\right\}-\left\{\frac{a}{\theta}+\frac{b(\theta t-1)}{\theta^{2}}+\frac{c\left[(\theta t-1)^{2}+1\right]}{\theta^{3}}\right\}
$$

Case.2.The inventory level having shortage:

In this period $\left[\mathrm{t}_{1}, \mathrm{~T}\right]$, the inventory level depends on demand and a fraction of demand is backlogged .The state of inventory during $\left[\mathrm{t}_{1}, \mathrm{~T}\right]$ form the differential equation:

$\frac{d I_{N}}{d t}+\theta I_{N}=\frac{-1}{1+\delta(T-t)}\left(\mathrm{a}+\mathrm{bt}+\mathrm{ct}^{2}\right), \mathrm{t}_{1} \leq \mathrm{t} \leq \mathrm{t}_{2},\left(\right.$ where $\left.\mathrm{T}=\mathrm{t}_{1}+\mathrm{t}_{2}\right) ;$

With boundary conditions $\mathrm{I}_{\mathrm{N}}(\mathrm{t})_{=} 0$ at $\mathrm{t}=\mathrm{t}_{1}$.

The solution of this system:

$\mathrm{I}_{\mathrm{N}}=\frac{1}{\delta}\left(a+b T+c T^{2}+\frac{b}{\delta}+\frac{c}{\delta}+\frac{2 T}{\delta}\right) \log \left[\frac{1+\delta(T-t)}{1+\delta\left(T-t_{1}\right)}\right]+\frac{1}{\delta}[b+2 c+2 T]\left(t-t_{1}\right)+\quad \frac{c}{\delta}\left[\left(t-t_{1}\right)\left\{\delta\left(t+t_{1}\right)-2 \delta-\right.\right.$ $2\}]$

\section{Inventory holding cost}

$$
\begin{aligned}
\mathrm{IC}_{\mathrm{H}}(\mathrm{t})= & \int_{0}^{t_{1}} h(t) e^{-r t} I_{P}(t) d t \\
= & \int_{0}^{t_{1}} h e^{-r t} I_{P}(t) d t, \\
\mathrm{IC}_{\mathrm{H}}(\mathrm{t})= & h\left\{\frac{a}{\theta}+\frac{b\left(\theta t_{1}-1\right)}{\theta^{2}}+\frac{\left.c\left(\theta t_{1}-1\right)^{2}+1\right]}{\theta^{3}}\right\}\left[\frac{\left(1-e^{\left.-r t_{1}\right)}\right)}{(r+\theta)}\right]-\left\{\frac{a}{\theta}\left[\frac{\left(1-e^{\left.-r t_{1}\right)}\right)}{r}\right]\right. \\
& +\frac{b}{\theta^{2}}\left[\left(\theta t_{1}-1\right) e^{-r t_{1}}-\frac{\theta}{r^{2}} e^{-r t_{1}}-\frac{1}{r}+\frac{\theta}{r^{2}}\right] \\
& +\frac{c}{\theta^{3}}\left[\left(\frac{1}{r}-\frac{2 \theta}{r^{2}}+\frac{2 \theta^{2}}{r^{3}}\right)-\left\{\left(t_{1}-1\right)^{2}+1\right\} \frac{e^{-r t_{1}}}{r}-\frac{2 \theta\left(t_{1} \theta-1\right) e^{-r t_{1}}}{r^{2}}+\frac{2 \theta e^{-r t_{1}}}{r^{3}}\right]
\end{aligned}
$$

\section{Backordered cost}

$\mathrm{C}_{\mathrm{B}}=\int_{t_{1}}^{T}\left(-I_{N}\right) e^{-r t} d t$ 
$\mathrm{C}_{\mathrm{B}}=-\int_{t_{1}}^{T} \quad \frac{1}{\delta}\left(a+b T+c T^{2}+\frac{b}{\delta}+\frac{c}{\delta}+\frac{2 T}{\delta}\right) \log \left[\frac{1+\delta(T-t)}{1+\delta\left(T-t_{1}\right)}\right] e^{-r t}+\frac{1}{\delta}[b+2 c+2 T]\left(t-t_{1}\right) e^{-r t}+\quad \frac{c}{\delta}[(t-$ $\left.\left.t_{1}\right)\left\{\delta\left(t+t_{1}\right)-2 \delta T-2\right\} e^{-r t}\right] d t$ $=\frac{1}{\delta^{2}}\left(a+b T+c T^{2}+\frac{b}{\delta}+\frac{c}{\delta}+\frac{2 T}{\delta}\right)\left\{\left[1+2 \delta\left(T-t_{1}\right)\right] \log \left[1+\delta\left(T-t_{1}\right)\right]\right\}+\frac{1}{2 \delta}[b+2 c+2 T](T-$

$\left.t_{1}\right)^{2}+\frac{c}{2 \delta}\left[\left(T-t_{1}\right)^{2}\left\{\delta\left(T+t_{1}\right)-2 \delta T-2\right\}\right]-\frac{c}{6}\left(T-t_{1}\right)^{3}-\frac{r}{\delta^{2}}\left(a+b T+c T^{2}+\frac{b}{\delta}+\frac{c}{\delta}+\frac{2 T}{\delta}\right)\left\{\frac{t_{1}}{\delta}+\frac{T}{\delta}+\right.$ $\left.\frac{1}{\delta^{2}}+\left(\frac{T^{2}}{\delta}+\frac{2 T}{\delta^{2}}+\frac{1}{\delta^{3}}\right) \log \left[1+\delta\left(T-t_{1}\right)\right]\right\}-\frac{r}{6 \delta}[b+2 c+2 T]\left(t_{1}{ }^{3}-2 T^{3}+3 T^{2} t_{1}\right)-\frac{r c}{12 \delta}\left\{\delta\left(3 t_{1}{ }^{4}-4 T t_{1}{ }^{3}-\right.\right.$ $\left.\left.11 T^{4}+12 t_{1} T^{3}\right)+12 t_{1}{ }^{2}-12 T^{2}\right\}$

\section{Lost sales cost}

$$
\begin{aligned}
& \mathrm{C}_{\mathrm{L}}=\int_{t_{1}}^{T}\left[1-\frac{1}{1+\delta(T-t)}\right]\left(\mathrm{a}+\mathrm{bt}+\mathrm{ct}^{2}\right) e^{-r t} d t \\
&\left.=\int_{t_{1}}^{T}\left\{\left[1-\frac{1}{1+\delta(T-t)}\right] \mathrm{a} e^{-r t}+\left[1-\frac{1}{1+\delta(T-t)}\right] e^{-r t} \mathrm{bt}+\left[1-\frac{1}{1+\delta(T-t)}\right] \mathrm{ct}^{2}\right) e^{-r t}\right\} d t \\
&=a\left[\left(T-t_{1}\right)-\frac{1}{\delta} \log \left\{1+\delta\left(T-t_{1}\right)\right\}-r\left\{\frac{\left(T^{2}-t_{1}{ }^{2}\right)}{2}+\frac{1}{\delta}\left(T-t_{1}\right)-\frac{1}{\delta}\left(T+\frac{1}{\delta}\right) \log \{1+\quad \delta(T-\right.\right. \\
&\left.\left.\left.\left.t_{1}\right)\right\}\right\}\right]+b\left[\left(T^{2}-t_{1}{ }^{2}\right)+\frac{1}{\delta}\left(T-t_{1}\right)-\frac{1}{\delta}\left(T+\frac{1}{\delta}\right) \log \left\{1+\delta\left(T-t_{1}\right)\right\}-\quad r\left\{\frac{\left(T^{3}-t_{1}{ }^{3}\right)}{3}+\frac{1}{2 \delta}\left(T^{2}-t_{1}{ }^{2}\right)+\right.\right. \\
&\left.\left.\frac{1}{\delta}\left(T+\frac{1}{\delta}\right)\left(T-t_{1}\right)-\frac{1}{\delta}\left(T+\frac{1}{\delta}\right)^{2} \log \left\{1+\delta\left(T-t_{1}\right)\right\}\right\}\right]+\quad c\left[\frac{\left(T^{3}-t_{1}{ }^{3}\right)}{3}+\frac{1}{2 \delta}\left(T^{2}-t_{1}{ }^{2}\right)+\frac{1}{\delta}\left(T+\frac{1}{\delta}\right)(T-\right. \\
&\left.t_{1}\right)-\frac{1}{\delta}\left(T+\frac{1}{\delta}\right)^{2} \log \left\{1+\delta\left(T-t_{1}\right)\right\}-\quad r\left\{\frac{\left(T^{4}-t_{1}{ }^{4}\right)}{4}+\frac{1}{3 \delta}\left(T^{3}-t_{1}{ }^{3}\right)+\frac{1}{\delta}\left(T+\frac{1}{\delta}\right)\left(T^{2}-t_{1}{ }^{2}\right)+\right. \\
& \frac{1}{\delta}(T+\left.\frac{1}{\delta}\right)^{2}\left(T-t_{1}\right)- \\
& \frac{1}{\delta}(T+\left.\left.\left.\frac{1}{\delta}\right)^{3} \log \left\{1+\delta\left(T-t_{1}\right)\right\}\right\}\right]
\end{aligned}
$$

\section{Purchase cost}

$$
\begin{aligned}
\mathrm{PC} & =(\text { Purchase cost per unit }) *(\text { Order quantity in one cycle }) \\
& =\mathrm{C}_{\mathrm{P}} * \mathrm{Q}_{\mathrm{o}}
\end{aligned}
$$

When $t=0$, the level of inventory is maximum and it is denoted by $I_{\max }\left[I_{P}(0)\right]$ then using equation (4)

$$
\mathrm{I}_{\max }=e^{\theta t_{1}}\left\{\frac{a}{\theta}+\frac{b\left(\theta t_{1}-1\right)}{\theta^{2}}+\frac{c\left[\left(\theta t_{1}-1\right)^{2}+1\right]}{\theta^{3}}\right\}-\left\{\frac{a}{\theta}-\frac{b}{\theta^{2}}+\frac{2 c}{\theta^{3}}\right\}
$$

The maximum backordered inventory is obtained at $t=t_{1}+t_{2}$, using equation (6)

$\mathrm{IB}_{\max }=-I_{N}\left(t_{1}+t_{2}\right)$

$$
=\frac{1}{\delta}\left\{\left(a+b T+c T^{2}+\frac{b}{\delta}+\frac{c}{\delta}+\frac{2 T}{\delta}\right) \log \left[1+\delta\left(T-t_{1}\right)\right]-\left(b+2 c+2 T\left(T-t_{1}\right)\right)-c\left(T-t_{1}\right)(\delta(T-\right.
$$

Thus the ordered size during total time interval $[0, \mathrm{~T}]$

$$
\left.\left.\left.t_{1}\right)+2\right)\right\}
$$

$$
\mathrm{Q}_{\mathrm{o}}=\mathrm{I}_{\max }+\mathrm{IB} \text { max }
$$

Now using the equations (10) and (11), we get

$$
\begin{aligned}
\mathrm{Q}_{\mathrm{o}}= & e^{\theta t_{1}}\left\{\frac{a}{\theta}+\frac{b\left(\theta t_{1}-1\right)}{\theta^{2}}+\frac{c\left[\left(\theta t_{1}-1\right)^{2}+1\right]}{\theta^{3}}\right\}-\left\{\frac{a}{\theta}-\frac{b}{\theta^{2}}+\frac{2 c}{\theta^{3}}\right\} \\
+ & \frac{1}{\delta}\left\{\left(a+b T+c T^{2}+\frac{b}{\delta}+\frac{c}{\delta}+\frac{2 T}{\delta}\right) \log \left[1+\delta\left(T-t_{1}\right)\right]\right. \\
& \left.-\left(b+2 c+2 T\left(T-t_{1}\right)\right)-c\left(T-t_{1}\right)\left(\delta\left(T-t_{1}\right)+2\right)\right\},
\end{aligned}
$$

Thus

$$
\mathrm{PC}=\mathrm{C}_{\mathrm{p}} * e^{\theta t_{1}}\left\{\frac{a}{\theta}+\frac{b\left(\theta t_{1}-1\right)}{\theta^{2}}+\frac{c\left[\left(\theta t_{1}-1\right)^{2}+1\right]}{\theta^{3}}\right\}-\left\{\frac{a}{\theta}-\frac{b}{\theta^{2}}+\frac{2 c}{\theta^{3}}\right\}
$$


$+\frac{1}{\delta}\left\{\left(a+b T+c T^{2}+\frac{b}{\delta}+\frac{c}{\delta}+\frac{2 T}{\delta}\right) \log \left[1+\delta\left(T-t_{1}\right)\right]-\left(b+2 c+2 T\left(T-t_{1}\right)\right)-c(T-\right.$ $\left.t_{1}\right)\left(\delta\left(T-t_{1}\right)+2\right)$,

\section{Ordering $\operatorname{cost} \mathrm{C}_{0}=\mathrm{A}$,}

\section{Total Cost}

Therefore the total cost per time unit is given by

$$
\begin{aligned}
& \mathrm{T}_{\mathrm{C}}\left(t_{1}, T\right)=\frac{1}{T}[\text { Ordering cost }+ \text { carring cost }+ \text { backordering cost } \\
& \text { + lost sale cost + purchase cost ] } \\
& =\frac{1}{T}\left[C_{O}+I C_{H}+C_{B}+C_{L}+P C\right] \text {, } \\
& \mathrm{T}_{\mathrm{C}}\left(t_{1}, T\right)=\frac{1}{T} A\left\{+\left\{\frac{a}{\theta}+\frac{b\left(\theta t_{1}-1\right)}{\theta^{2}}+\frac{\left.c\left(\theta t_{1}-1\right)^{2}+1\right]}{\theta^{3}}\right\}\left[\frac{\left(1-e^{\left.-r t_{1}\right)}\right)}{(r+\theta)}\right]-\left\{\frac{a}{\theta}\left[\frac{\left(1-e^{\left.-r t_{1}\right)}\right)}{r}\right]\right.\right. \\
& +\frac{b}{\theta^{2}}\left[\left(\theta t_{1}-1\right) e^{-r t_{1}}-\frac{\theta}{r^{2}} e^{-r t_{1}}-\frac{1}{r}+\frac{\theta}{r^{2}}\right]+\frac{c}{\theta^{3}}\left[\left(\frac{1}{r}-\frac{2 \theta}{r^{2}}+\frac{2 \theta^{2}}{r^{3}}\right)\right. \\
& \left.-\left\{\left(t_{1}-1\right)^{2}+1\right\} \frac{e^{-r t_{1}}}{r}-\frac{2 \theta\left(t_{1} \theta-1\right) e^{-r t_{1}}}{r^{2}}+\frac{2 \theta e^{-r t_{1}}}{r^{3}}\right] \\
& +\frac{1}{\delta^{2}}\left(a+b T+c T^{2}+\frac{b}{\delta}+\frac{c}{\delta}+\frac{2 T}{\delta}\right)\left\{\left[1+2 \delta\left(T-t_{1}\right)\right] \log \left[1+\delta\left(T-t_{1}\right)\right]\right\}+\quad \frac{1}{2 \delta}[b+2 c+ \\
& 2 T]\left(T-t_{1}\right)^{2}+\frac{c}{2 \delta}\left[\left(T-t_{1}\right)^{2}\left\{\delta\left(T+t_{1}\right)-2 \delta T-2\right\}\right]-\quad \frac{c}{6}\left(T-t_{1}\right)^{3}-\frac{r}{\delta^{2}}\left(a+b T+c T^{2}+\frac{b}{\delta}+\right. \\
& \left.\frac{c}{\delta}+\frac{2 T}{\delta}\right)\left\{\frac{t_{1}}{\delta}+\frac{T}{\delta}+\frac{1}{\delta^{2}}+\left(\frac{T^{2}}{\delta}+\frac{2 T}{\delta^{2}}+\quad \frac{1}{\delta^{3}}\right) \log \left[1+\delta\left(T-t_{1}\right)\right]\right\}-\frac{r}{6 \delta}[b+2 c+2 T]\left(t_{1}{ }^{3}-2 T^{3}+\right. \\
& \left.3 T^{2} t_{1}\right)-\quad \frac{r c}{12 \delta}\left\{\delta\left(3 t_{1}{ }^{4}-4 T t_{1}{ }^{3}-11 T^{4}+12 t_{1} T^{3}\right)+12 t_{1}{ }^{2}-12 T^{2}\right\}+ \\
& a\left[\left(T-t_{1}\right)-\frac{1}{\delta} \log \left\{1+\delta\left(T-t_{1}\right)\right\}-r\left\{\frac{\left(T^{2}-t_{1}^{2}\right)}{2}+\frac{1}{\delta}\left(T-t_{1}\right)-\frac{1}{\delta}\left(T+\frac{1}{\delta}\right) \log \{1+\quad \delta(T-\right.\right. \\
& \left.\left.\left.\left.t_{1}\right)\right\}\right\}\right]+b\left[\left(T^{2}-t_{1}{ }^{2}\right)+\frac{1}{\delta}\left(T-t_{1}\right)-\frac{1}{\delta}\left(T+\frac{1}{\delta}\right) \log \left\{1+\delta\left(T-t_{1}\right)\right\}-\quad r\left\{\frac{\left(T^{3}-t_{1}{ }^{3}\right)}{3}+\frac{1}{2 \delta}\left(T^{2}-\right.\right.\right. \\
& \left.\left.\left.t_{1}{ }^{2}\right)+\frac{1}{\delta}\left(T+\frac{1}{\delta}\right)\left(T-t_{1}\right)-\frac{1}{\delta}\left(T+\frac{1}{\delta}\right)^{2} \log \left\{1+\delta\left(T-t_{1}\right)\right\}\right\}\right]+\quad c\left[\frac{\left(T^{3}-t_{1}{ }^{3}\right)}{3}+\frac{1}{2 \delta}\left(T^{2}-t_{1}{ }^{2}\right)+\right. \\
& \frac{1}{\delta}\left(T+\frac{1}{\delta}\right)\left(T-t_{1}\right)-\frac{1}{\delta}\left(T+\frac{1}{\delta}\right)^{2} \log \left\{1+\delta\left(T-t_{1}\right)\right\}-\quad r\left\{\frac{\left(T^{4}-t_{1}^{4}\right)}{4}+\frac{1}{3 \delta}\left(T^{3}-t_{1}{ }^{3}\right)+\frac{1}{\delta}\left(T+\frac{1}{\delta}\right)\left(T^{2}-\right.\right. \\
& \left.\left.\left.t_{1}{ }^{2}\right)+\frac{1}{\delta}\left(T+\frac{1}{\delta}\right)^{2}\left(T-t_{1}\right)-\quad \frac{1}{\delta}\left(T+\frac{1}{\delta}\right)^{3} \log \left\{1+\delta\left(T-t_{1}\right)\right\}\right\}\right]+\mathrm{C}_{\mathrm{p}} \quad * e^{\theta t_{1}}\left\{\frac{a}{\theta} \quad+\frac{b\left(\theta t_{1}-1\right)}{\theta^{2}}\right. \\
& \left.+\frac{c\left[\left(\theta t_{1}-1\right)^{2}+1\right]}{\theta^{3}}\right\}- \\
& \left\{\frac{a}{\theta}-\frac{b}{\theta^{2}}+\frac{2 c}{\theta^{3}}\right\}+\frac{1}{\delta}\left\{\left(a+b T+c T^{2}+\frac{b}{\delta}+\frac{c}{\delta}+\frac{2 T}{\delta}\right) \log \left[1+\delta\left(T-t_{1}\right)\right]-(b+\quad 2 c+\right. \\
& \left.2 T\left(T-t_{1}\right)\right)-c\left(T-t_{1}\right)\left(\delta\left(T-t_{1}\right)+2\right),
\end{aligned}
$$

The necessary condition for the total cost per time unit to be minimize is

Provided,

$$
\frac{\partial T_{C}}{\partial t_{1}}=0 \text { and } \frac{\partial T_{C}}{\partial t_{2}}=0
$$

\section{Alogrithm}

$$
\left(\frac{\partial^{2} T_{c}}{\partial t_{1}^{2}}\right)\left(\frac{\partial^{2} T_{c}}{\partial t_{2}^{2}}\right)-\left(\frac{\partial^{2} T_{c}}{\partial t_{1} t_{2}}\right)>o
$$

To find out the solution following algorithm used

Step1 Find derivative $\frac{\partial T_{c}}{\partial t_{1}}=0, \frac{\partial T_{C}}{\partial t_{2}}=0, \frac{\partial^{2} T_{c}}{\partial^{2} t_{1}}, \frac{\partial^{2} T_{c}}{\partial^{2} t_{2}}, \frac{\partial^{2} T_{c}}{\partial t_{1} t_{2}}$

Step2 Solve (15) for $t_{1}$ and $t_{2}$ where $T=t_{1}+t_{2}$

Step3 Find total cost from equation (14)

\section{Conclusion}

In this paper, we developed a model for deteriorating item with inflation, quadratic demand and partial backlogging and give analytical solution of the model that minimize the total inventory cost. The deterioration 
factor taken into consideration in the present model, as almost all items undergo either direct spoilage or physical decay in the course time, deterioration is natural feature in the inventory system. The model is very practical for the industries in which the demand rate is depending upon the time and holding cost is constant with inflation. This model can further be extended by taking more realistic assumptions such as finite replenishment rate, probabilistic demand rate, variable deterioration rate etc.

\section{Numerical Solution}

Consider an inventory system with the following parameter in proper unit $\mathrm{A}=2500, \mathrm{~h}=0.5, \mathrm{C}=$ $4, \mathrm{C}_{\mathrm{B}}=12, \mathrm{C}_{1}=15, \delta=8, \mathrm{a}=25, \mathrm{~b}=40, \mathrm{c}=40, \theta=0.005$ and parameter $\mathrm{r}=.005$. The computer out put of the program by using maple mathematical software is $t_{1}=4.12, t_{2}=0.03$ and TC $=1179$. i.e. the value of $t_{1}$ at which the inventory level become zero is 4.12 unit and shortage period is 0.03 unit. The effect of changes in the parameter of the inventory model also can be study.

\section{References}

[1]. Abad, P. (1996). Optimal pricing and lot-sizing under conditions of perishability and partial backordering. Management Science 42,1093-1104.

[2]. Abad, P. (2001). Optimal price and order-size for a reseller under partial backlogging. Computers and Operation Research 28,5365.

[3]. Alamri, A. and Balkhi, Z. (2007). The effects of learning and forgetting on the optimal production lot size for deteriorating tems with time varying demand and deterioration rates. International Journal of Production Economics, 107,125-138.

[4]. Buzacott, J.A., 1975. Economic order quantities with inflation. Operational Research Quarterly, 26: 553-558.

[5]. Chang, C.T. (2004): An EOQ model with deteriorating items under inflation when supplier credits linked to order quantity. International Journal of Production Economics, 88, 307-316.

[6]. Chang, H. and Dye, C. (1999). An EOQ model for deteriorating items with time varying demand and partial backlogging.Journal of the Operational Research Society, 50,1176-1182.

[7]. Dave, U. and Patel, L. (1981). (T, si)-policy inventory model for deteriorating items with time proportional demand. Journal of Operational Research Society, 32,137-142.

[8]. Dye, C. (2007a). Determining optimal selling price and lot size with a varying rate of deterioration and exponential partial backlogging. European Journal of Operational Research,181,668-678.

[9]. Dye, C. (2007b). Joint pricing and ordering for a deteriorating inventory with partial backlogging. Omega, 35(2),184-189.

[10]. Dye, C., Ouyang, L., and Hsieh, T. (2007). Deterministic inventory model for deteriorating items with capacity constraint and timeproportional backlogging rate. European Journal of Operational Research, 178(3),789-807.

[11]. Ghare, P. and Schrader, G. (1963). A model for an exponentially decaying inventory. Journal of Industrial Engineering,14,238-243.

[12]. Goyal, S. and Giri, B. (2001). Recent trends in modeling of deteriorating inventory. European Journal of Operational Research, $134,1-16$.

[13]. Harris, F. (1915). Operations and cost. Chicago: AW Shaw Co.

[14]. He, Y., Wang, S., and Lai, K. (2010). An optimal production-inventory model for deteriorating items with multiple-market demand. European Journal of Operational Research, 203(3),593-600.

[15]. Hung, K. (2011). An inventory model with generalized type demand, deterioration and backorder rates. European Journal of Operational Research, 208(3):239-242.

[16]. Jalan, A., Giri, R., and Chaudhary, K. (1996). EOQ model for items with weibull distribution deterioration shortages and trended demand. International Journal of System Science,27:851-855.

[17]. Liao, J. (2008). An EOQ model with non instantaneous receipt and exponential deteriorating item under two-level trade credit. International Journal of Production Economics, 113:852-861.

[18]. Mandal, B. (2010). An EOQ inventory model for Weibull distributed deteriorating items under ramp type demand and shortages. Opsearch, 47(2):158-165.

[19]. Mishra, V. and Singh, L. (2011). Inventory model for ramp type demand, time dependent deteriorating items with salvage value and shortages. International Journal of Applied Mathematics \& Statistics, 23(D11):84-91.

[20]. Misra, R.B., 1979. A note on optimal inventory management under inflation. Naval Research Logistics Quarterly, 26: 161-165.

[21]. Pareek, S., Mishra, V., and Rani, S. (2009). An Inventory Model for time dependent deteriorating item with salvage value and shortages. Mathematics Today, 25:31-39.

[22]. Roy, A. (2008). An inventory model for deteriorating items with price dependent demand and time varying holding cost. Advanced Modeling and Optimization, 10:25-37.

[23]. Shah, N. and Shukla, K. (2009). Deteriorating inventory model for waiting time partial backlogging. Applied Mathematical Sciences, 3:421-428.

[24]. Skouri, K., Konstantaras, S., and Ganas, I. (2009). Inventory models with ramp type demand rate, partial backlogging and weibull deterioration rate. European Journal of Operational Research, 192:79-92.

[25]. Teng, J., Ouyang, L., and Chen, L. (2007). A comparison between two pricing and lot-sizing models with partial backlogging and deteriorated items. International Journal of Production Economics, 105:190-203.

[26]. Whitin, T. (1957). The Theory of Inventory Management.Princeton University Press, New Jersey, 2nd edition.

[27]. Wilson, R. (1934). A scientific routine for stock control.Harvard Business Review, 13:116-128.

[28]. Wu, O. and Cheng (2005). An inventory model for deteriorating items with exponential declining demand and partial backlogging. Yugoslav Journal of Operations Research,15(2):277-288.IJMSEM.

[29]. Yadav,R.K and Trivedi,R.(2013) A multi - echolen inventory models for deteriorating with partial backlogging ,International journal of applied science and technology, 24-31.

[30]. Yadav,R.K and Yadav,P.(2013). Volume flexibility in production model with cubic demand rate and weibull deterioration with partial backlogging IOSR Journal of Mathematics 6(4):29-34.

[31]. Yadav,R.K. and Devi,P. (2013).Development of an inventory model with volume flexibility, random deterioration and increasing exponentially demand rate. IOSR Journal of Mathematics VOL.X(X).

[32]. Yang, H.L. (2006): Two-warehouse partial backlogging inventory models for deteriorating items under inflation. International Journal of Production Economics, 103(1), 362-370. 